\title{
Durability of metal structures under quasi-static load
}

\author{
Dmitriy Ogorelkov², Vladimir Mironov ${ }^{1,2}$, and Olga Lukashuk ${ }^{2, *}$ \\ ${ }^{1}$ Institute of Engineering Science, RAS (Ural Branch), Yekaterinburg, Russia \\ ${ }^{2}$ Ural Federal University, Yekaterinburg, Russia
}

\begin{abstract}
Failure of materials and structures is one of unresolved problems of mechanics. This paper offers an approximate approach to assessing durability of products on the basis of a mechanical experiment. The experiment represents the fatigue process as a transition of a plastic material into its brittle state. A simplified physical model - which could be used to build a mathematical model of fatigue process - hangs on a local transition of a plastic material into its brittle state. The calculation methodology includes both an original part on cyclic degradation of material strength and correlations based on experiments and checked by design routines. Two approaches to calculating the durability of a randomly-loaded object are compared: using the equation of cyclic degradation of strength and the rule of linear summation of fatigue damages. The results obtained are useful for improving methodologies of calculating service life or durability of structures.
\end{abstract}

\section{Introduction}

In-process loads on bearing structures of mining and transporting machines, as a rule, are random by their nature. When service life is calculated, a random process is reduced to a stationary process, equivalent in terms of damage. It is achieved with the help of the linear hypothesis of fatigue damage summation, which cannot be physical justified and is followed by estimation of durability on the basis of a fatigue curve with a preset probability of failure. The number of cycles prior to failure obtained like that often disagrees quite considerably with data on failures of metallic structures in operation.

From the viewpoint of physical reliability, the methods of design should be based on the research into degradation processes in structural materials. Many processes which take place in the whole volume of a material lead to changes in the diagram of static deformation, which is reflected in rheological equations. It was just how such theories as the creep theory, long-term strength theory, hydrogen embrittlement theory and many others were developed [1-3]. Fatigue damages accumulate in a workpiece's surface layer of up to 200 microns in depth and get localized in areas of 50-100 microns [4,5] - which requires special testing methods to be utilized. Fatigue-testing of typical specimens in

\footnotetext{
* Corresponding author: oldim96@mail.ru
} 
reveals no change in the static diagram of deformation [6,7]. The methods which could be used to calculate durability and remaining life of structural elements, while taking a specified level of localization into consideration, are just being developed. This paper offers an approximate approach to assessing durability of products on the basis of a mechanical experiment. The experiment represents the fatigue process as a transition of a plastic material into its brittle state $[8,9]$.

Physically, the mechanism of fatigue is related to cycle-by-cycle displacement of dislocations, formation of critical dislocation structures, initiation and development of fatigue cracks.

A simplified physical model - which could be used to build a mathematical model of fatigue process - hangs on a local transition of a plastic material into its brittle state.

Such transitions are recorded in fatigue tests of smaller thin-wall specimens, which allow to draw a complete static diagram with a branch descending to zero [9,10].

Besides available plasticity, determined by the length of a diagram, such characteristics representative of a material as the modulus of elasticity, ultimate strength, modulus of brittleness diminish. The force-based approach uses the ultimate strength $S_{B}$ as a parameter representative of the fatigue process. In the case of a regular loading, it becomes a function of maximal stress in a cycle $\sigma_{M}$ and the number of loading cycles, and is approximated via a exponential function $S_{B}\left(\sigma_{M} ; n\right)=S_{B 0}-k_{S} n^{m}$, where $S_{B 0}$ is the ultimate strength of an untrained material, $\mathrm{m}$ is an experimental constant, close to 2 for the materials studied. The coefficient $k_{S}$ could be determined on the condition of a specimen failing due to fatigue, when its ultimate strength falls to the level of maximal stress in a cycle

$$
S_{B}\left(\sigma_{M} ; N_{R}\right)=\sigma_{M}
$$

where $N_{R}$ is durability according to a fatigue curve drawn on the condition of a crack initiated and with $R$ as a asymmetry coefficient.

The evolutionary equation of multicycle fatigue takes the following form

$$
S_{B}\left(\sigma_{M} ; n\right)=S_{B 0}-\frac{S_{B 0}-\sigma_{M}}{N_{R}^{m}} n^{m} .
$$

Expression (2) agrees with the equations of the elasticity theory, which are used to determine the stress-strain state of a metallic structure in the region of multicycle fatigue.

The current cyclic state of a material is determined by its ultimate strength, which is evaluated by formula (2). The criterion of failure (1) is generalized on the basis of nonstationary loading [9] in the form of a rule defining the intersection of the kinetic curve and the level of maximal stress. According to (2), the damaging action of a cycle of stresses depends on its position in the spectrum of loads, which excludes rearrangement of cycles in the spectrum and, therefore, application of correlation and spectral analysis.

For a random spectrum of loads and on the basis of the linear hypothesis of fatigue damage summation, the predicted durability $N_{L}$ is determined on the following condition

$$
\sum_{i=1}^{N_{L}} n_{i} / N_{i}=1
$$


Thus, the goal of the research was to compare two approaches to calculating the durability of an object randomly loaded: using the equation of cyclic degradation of strength (2) and the rule of linear summation of fatigue damages (3).

\section{Measure of cyclic material damage}

The measure of cyclic damage from the linear hypothesis $\omega_{L}=n / N$ is not physically justified, but it allows to rearrange cycles in the general spectrum, reduce them using various coefficients of asymmetry, and so on. For example, equality of damages after a certain number of cycles with a different coefficient of asymmetry could be reduced to equality of durabilities. Then, taking (2) into account, an initial cycle parameterized by $\sigma_{i}, R_{i}$ would be reduced to a symmetrical cycle parameterized by $\sigma_{r i}, R=-1$ via a known formula [11]

$$
\sigma_{r i}=\sigma_{i} \frac{\sigma_{-1 K}}{\sigma_{R K i}}
$$

where, for a tabular value of the endurance limit $\sigma_{-1 K}$, the endurance limit $\sigma_{R K}$, for an arbitrary cycle, is determined by an experimental expression

$$
\sigma_{R K}=\frac{2 \sigma_{-1 K}}{1-R+\psi_{K}(1+R)},
$$

where $\psi_{K}$ is coefficient of sensitivity to the asymmetry of a cycle, which is usually assumed to be equal 0 for welded structures.

The monograph [8] analyzes the cyclic damage $\omega_{C}$ of a model specimen from the viewpoint of structurally-phenomenological approach, assessing it via a relative number of structure elements fractured due to fatigue. An important expression was formulated, which connects the structural damage $\omega_{C}$ with a macro-parameter, namely, the ultimate strength

$$
S_{B}\left(\sigma_{M}, \mathrm{n}\right)=\left(1-\omega_{C}\right) S_{B 0} \cdot
$$

Expression (2) is used to approximate an experimental kinetic curve recorded from a breaking test run on special trained specimens [9]. Substituting (2) into (6), an expression in the form of Koten-Dolan could be obtained

$$
\omega_{C}=\frac{S_{B 0}-\sigma_{M}}{S_{B 0}}\left(\frac{n}{N\left(\sigma_{M}\right)}\right)^{m} .
$$

Expression (2) is formed on the assumption that fracture of a specimen occurs when the loading line intersects with the function of ultimate strength, which means $\omega_{C} \in\left[0, \omega_{C R}\right]$.

The damage at the moment of fracture (when $n=N$ ) is not equal 1: $\omega_{C R}=1-\frac{\sigma_{M}}{S_{B 0}}$.

Let us define a relative damage as the expression of $\overline{\omega_{C}}=\frac{\omega_{C}}{\omega_{C R}}=\left(\frac{n}{N}\right)^{m}$. 
Assuming the principle of equality between relative damages of the initial and reduced cycles to be correct, and taking $m=$ const, $\alpha=$ const, we get exactly the expression (4), which cannot be done using the expression (7). So, for further estimation of reduced stresses, expression (4) will be utilized.

\section{Methodology of calculating durability of bridge girders for overhead cranes}

The calculation methodology includes both an original part on cyclic degradation of material strength and correlations based on experiments and checked by design routines. With the goal of assessing comparatively the durability of a girder in mind, we consider quasi-random loading of a metallic structure within a specified variation range of maximal stresses in a cycle. The random nature of loading during the stages of starting and braking the hoisting mechanism of a crane is caused by mainly random values of the weight of a bulk load in the grab and acceleration of starting-braking operational modes.

The first stage of the calculation is aimed at determining the stress-strain state of a bridge girder using the method of finite elements and hot points with stress concentrators. In the example, it is the point in the lower chord of the girder, near the diaphragm close to the dangerous section, where welded joints intersect. In engineering design, it is a common practice to factor in only normal stresses when the durability of a crane is estimated [11]. The maximal stress for a filled bucket and the stress in the girder under no load are found.

The second stage focuses on randomizing maximal stresses within the range of their variation set in the previous stage.

The third one realizes the algorithm of durability calculation. For each cycle of stresses with a specified $\sigma_{M}$, the asymmetry coefficient $R$, tabular endurance limit $\sigma_{-1 K}$ and endurance limit $\sigma_{R K}$ (5) are evaluated. The cycle is reduced to a symmetrical one (4), and the durability $N$ (from equation (2) for a specified $\alpha$ [12]), damage on the linear hypothesis $\omega_{L}=1 / N$ and current ultimate strength (from formula (1) adapted to random loading) are estimated. If the current ultimate strength is higher than the maximal stress of the next cycle, transition is made to a new kinetic curve on the basis of the equation of cyclic damage (7). A relatively simple mathematical formalization used to describe the degradation process allows to run cycle-by-cycle computation of the damage just for a small portion of computer time.

The fourth stage begins with plotting curves for ultimate strength and (or) damages, which would allow to determine and compare the durabilities required. Now, parametrical analysis is also possible when there are statistical data on distribution of initial values of ultimate strength and fatigue available.

\section{Example of calculating durability of the bridge girder of a crane}

The first stage. Calculated stresses at a danger point of the bridge girder of an unloaded crane were equal to $74 \mathrm{MPa}$, for a filled grab - $176 \mathrm{MPa}$, and for the minimal filling - 135 $\mathrm{MPa}$ [13]. Thus, the volume of load, as a random value, spurs random maximal stresses within a hoisting cycle, which vary in the range of $\sigma_{M} \in[135,176] \mathrm{MPa}$. The lowest value of the cycle of stresses $(74 \mathrm{MPa})$ remains unchanged. 
The second stage. The sequence of maximal stresses in a cycle was calculated in the Python programming environment. The spectrum of stresses obtained had been repeated until a certain criterion of fatigue fracture was reached. Besides, in another variant of the same calculation, after each 200-th cycle, the spectrum above was supplemented with one additional cycle of $220 \mathrm{MPa}$ stresses. It was done to estimate the impact which rare but value-wise significant cycles of peak stresses had on the durability calculated. Equations should be centred and should be numbered with the number on the right-hand side.

The desired durability has only a conditional importance. To make a more precise prediction, one needs to acquire data on actual utilization of a crane, which is available from logs of a special recorder [14]. At the same time, using this data in methodologies which are based on the hypothesis of linear summation of fatigue damages and allow to calculate total service life or remaining life [15], one could end up with an incorrect prediction.

To fulfill the third stage, it was necessary to come up with an algorithm for calculating the durability life based on several variants of the task in question and realize it in a software developed in Python by the authors. The endurance limit in a symmetric cycle for a structure of 8-th group [11] is expressed as $\sigma_{-1 K}=36 \mathrm{M \Pi a}$ for a basic service life of $2 \cdot 10^{6}$ cycles. The exponent in relation (2), which determines the degradation rate for the strength of a material, was picked to serve as an indicator in the range of $[0,5 ; 4]$. The fatigue curve index for 09G2S steel is defined as $\alpha=5,3$ [12]. The testing of specimens aimed to plot the kinetic curve (2) was carried out in a pulsing cycle. Therefore, in calculations of the equivalent stress $\sigma_{r i}$ by means of (4), the right part of the equation was multiplied by two, keeping up with the conditions specified above.

The fourth stage. The results of calculations for the process of loading with a random maximal stress (Fig. 1,a) and via the formula and criterion (3) helped to relate the cyclic damage to the number of cycles (line 1 in Fig. 1,a).
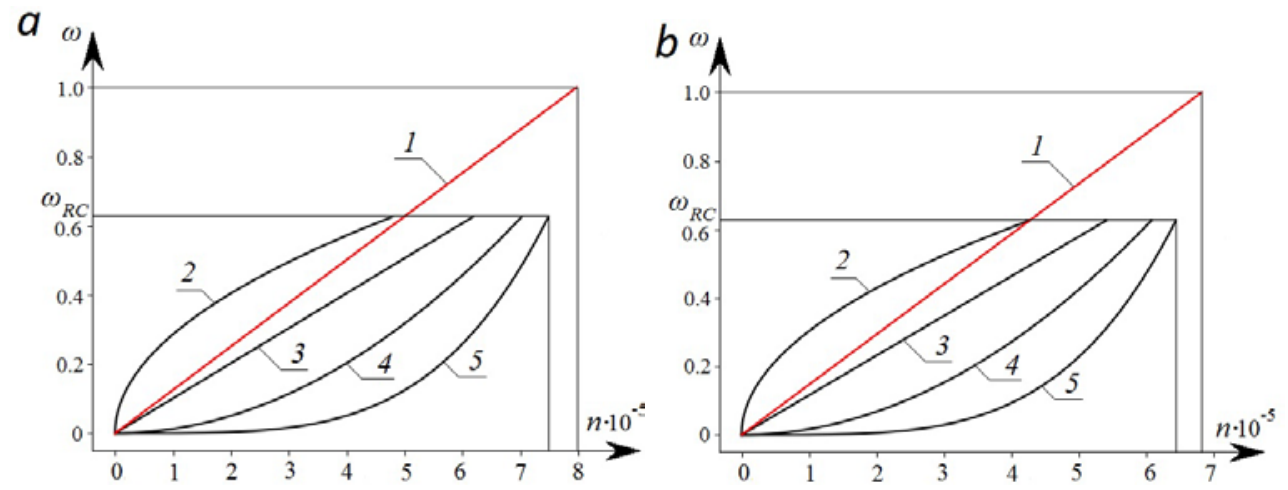

Fig. 1. Calculated damage: a) without peak overloads; b) when rare peak overloads are present in the spectrum

1 - on linear hypothesis, 2, 3, 4 and 5 for $m=0,5 ; m=1 ; m=2$ and $m=4$, correspondingly.

The durability life calculated using the linear summation of fatigue damages was 798000 cycles. The accumulation of damages from formula 2 and generalized criterion of fatigue fracture (1) is illustrated by curves 2-5 in Fig. 1, $a$, parameterized with a value of the $m$ constant. In the second case, the durability predicted is lower for any value of $m$ used (that is even when $m=1$ ), becoming closer to the linear summation for $m \rightarrow \infty$, when material properties remain unchanged until the failure. Specific values of the calculated durability $N_{*}$ is provided in the first row of Table 1 . 
Table 1. Values of the calculated durability $N_{*}$.

\begin{tabular}{|c|c|c|c|c|c|}
\hline $\boldsymbol{m}$ & $\mathbf{0 , 5}$ & $\mathbf{1}$ & $\mathbf{2}$ & $\mathbf{4}$ & $\begin{array}{c}\text { linear } \\
\text { hypothesis }\end{array}$ \\
\hline $\begin{array}{c}N^{*}, 10^{-3} \text { without } \\
\text { overloads }\end{array}$ & 478,7 & 618,2 & 702,4 & 748,7 & 798 \\
\hline $\begin{array}{c}N^{*}, 10^{-3} \text { with } \\
\text { overloads }\end{array}$ & 384 & 479,3 & 534,5 & 564,1 & 595,2 \\
\hline
\end{tabular}

The second row in Table 1 shows the results of parallel calculation of durability when peak overloads are present in the spectrum, with Fig. 1, $b$ demonstrating related calculated curves of damage. Introducing less than 50000 peak overloads into the spectrum of loading lead to substantial reduction in durability life, regardless of the calculation method.

\section{Conclusion}

Comparing the deterministic calculations cited here, one should note that introducing a physical criterion of fatigue fracture (1) significantly impacted the prediction given on fatigue life, and not in terms of its increase. More definite conclusions could be drawn after generalization of the approach and the line of experimental checking in terms of probability statistics. The computer time required to make a cycle-by-cycle summation of fatigue damages turned out to be quite small, even with a typical PC being used for the purposes. Similar tests on specimens and a computation experiment could be run in a factory lab or design-engineering department. The methodology based on a reproductible mechanical experiment on cyclic degradation and full-scale testing of welded joints results in a conservative prediction of the durability of a metallic structure and could well become a part of design routines.

The research was done as a part of a government order on the topic № 0391-2016-0001.

\section{References}

1. Y.N. Rabotnov, Creep in Structural Elements (Science, Moscow, 1955, 752 p.)

2. I.I. Goldenblatt, V.L. Bazhenov, V.A. Kopnov, Long-Term Strength in Machine Building (Machine Building, Moscow, 1977, 248 p.)

3. G.V. Karpenko, R.I. Kripyatkevich, Hydrogen Impact on Steel Properties (Metallurgy Publishing, Moscow, 1962, 192 p.)

4. T.Y. Yakovleva, Local Plastic Deformation and Fatigue of Metals (Scientific Thinking, Kiev, 2003, 238 p.)

5. V.I. Mironov, S.Y. Mitrpolskaya, Service Life and Diagnostics of Materials and Structures (Institute of Engineering Science, Yekaterinburg, 2009, pp. 97)

6. V.F. Terentiev, Fatigue Strength of Metals and Alloys (Intermetals Engineering, Moscow, 2002, 288 p.)

7. G.V. Uzhik (Ed.), Fatigue and Endurance of Metals: Collected Papers (Foreign Literature Publishing, Moscow, 1963, 487 p.)

8. V.V. Struzhanov, V.I. Mironov, Strain-Softening of Materials in Structural Elements (Ural Branch of Russian Academy of Science Publishing, Yekaterinburg, 1995, 190 p.)

9. I.G. Emeliyanov, V.I. Mironov, Durability of Shell Structures (Ural Branch of Russian Academy of Science Publishing, Yekaterinburg, 2012, 217 p.) 
10. V.I. Mironov, O.A. Lukashuk, D.I. Vichuzhanin, Solid State Phenom. J., 265, pp. 815820 (2017)

11. S.A. Sokolov, Structural Mechanics and Metallic Structures of Machines (Polytechnics, Saint Petersburg, 2011, 450 p.)

12. V.T. Trotschenko, L.A. Sosnovskiy, Fatigue Resistance of Metals and Alloys. Reference Book, 1 (Scientific Thinking, Kiev, 1987, 510 p.)

13. V.E. Dusie, Y.V. Navarskiy, V.P. Zhegulskiy, Calculation and Design of Bridge Crane Metallic Structures (UGTU-UPI, Ekaterinburg, 2007)

14. RD-10-399-01. Specifications of Parameter Recorders for Load-lifting Cranes (State Municipal Technical Control of Russian Federation, Moscow, 2001, 15 p.)

15. RD-24-112-5R. Guiding Document on Assessment of Remaining Service Life for Overhead-type Cranes (National Research Institute for Lifting and Transporting Engineering, Moscow, 2002, 23 p.) 